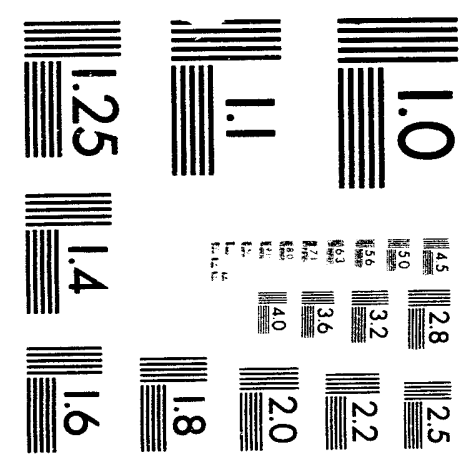




\section{ב.}
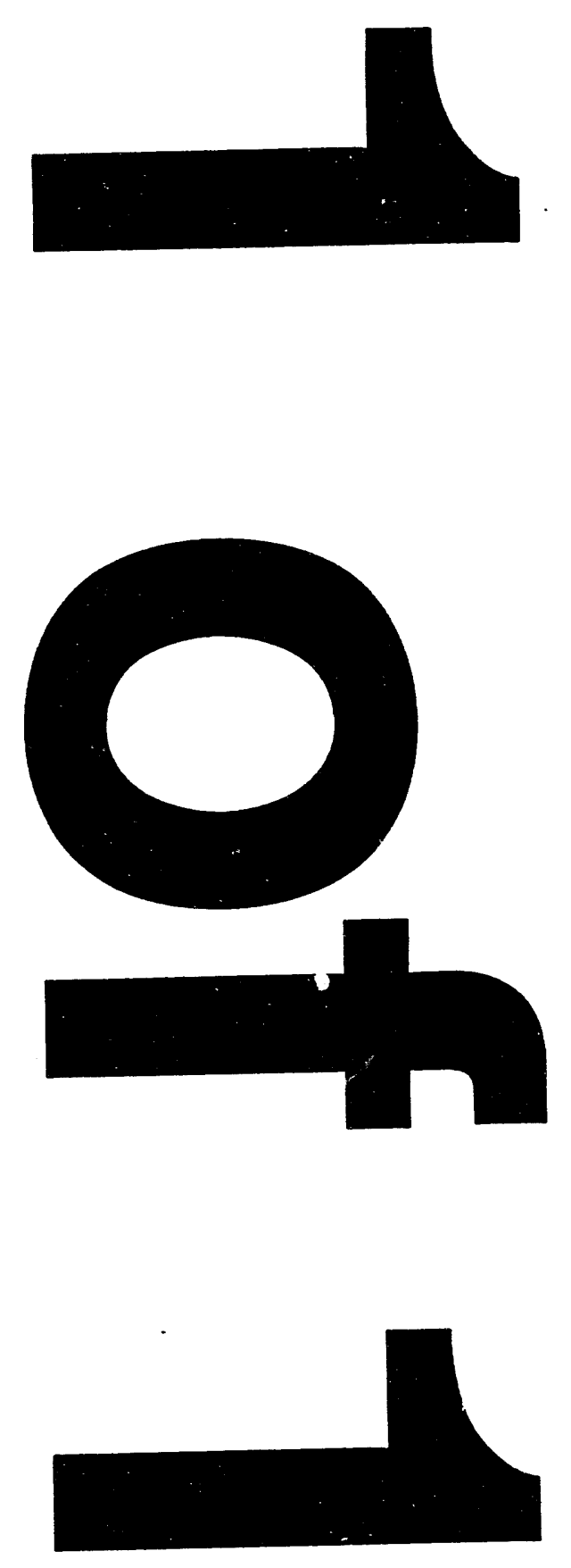

$\longrightarrow$ 
LA-UR- $93-4340$
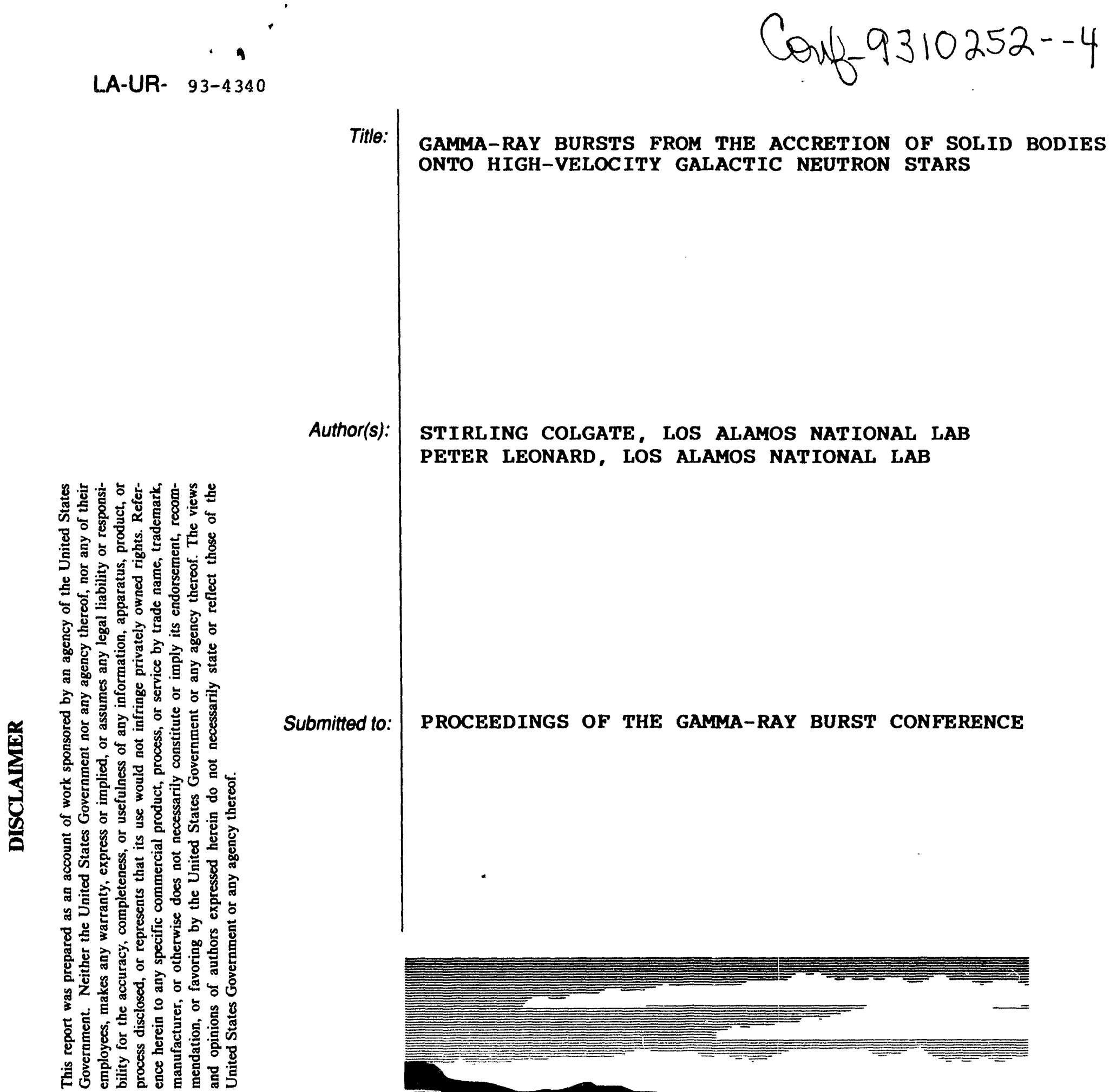

Author(s):

STIRL ING COLGATE, LOS ALAMOS NATIONAL LAB

PETER LEONARD, LOS ALAMOS NATIONAL LAB

Submitted to:

PROCEEDINGS OF THE GAMMA-RAY BURST CONFERENCE

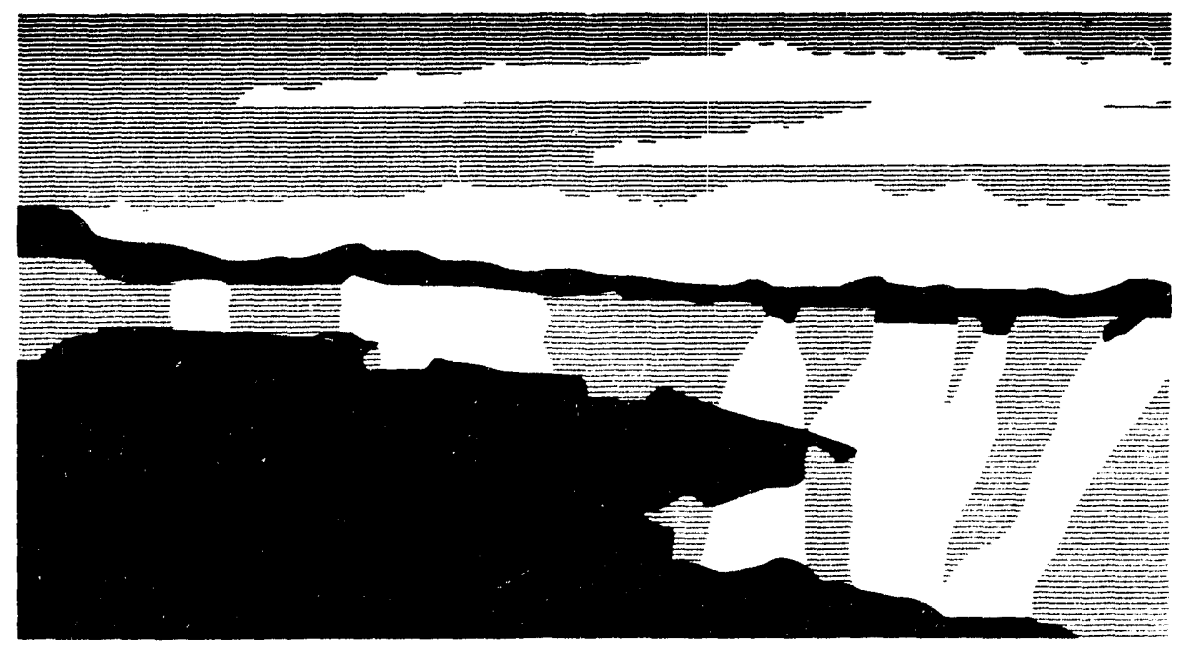

Los Alamos National Laboratory, an aftirmative action/equal opportunity emplobyer, is operated by the University of Calitornia for the U.S. Department of Energy under contract W.7405-ENG-36. By acceptance of this articte, the publisher recognizes that the U.S. Government retains a nonexclusive, royalty-free license to publish or reproduce the published form of this contribution, or to allow others to do so, for U.S. Government purposes. The Los Alamos National Laboratory requests that the publisher identity this article as work performed under the auspices of the U.S. Department of Entrgy.

$$
\text { Fingy in }
$$

Form No. 836 A5 ST $262910 / 91$ 


\title{
GAMMA-RAY BURSTS FROM THE ACCRETION OF SOLID BODIES ONTO HIGH-VELOCITY GALACTIC NEUTRON STARS
}

\author{
Stirling A. Colgate and Peter J. T. Leonard \\ T-6. MS B275. Los Alamos National Laboratory, Los Alamos. NM 87545
}

ABSTRAC'T

We propose a simple model for the gamma-ray bursts based on high-velocity Galactic neutron stars that have accretion disks. The latter are formed from a mixture of material from the supernova shell and that ablated from a pre-supernova binary companion. Accretion onto the neutron star from this disk when the disk is still largely gaseous may result in a soft gamma-ray repeater phase. Much later, after the neutron star has moved away from its birthplace. solid bodies form in the disk, and some are perturbed into hitting the neutron star to create gamma-ray bursts. This model makes several predictions that are consistent with the observations. The observed combination of a high degree of isotropy on the sky coupled with the observed value of $\left\langle V / V_{\max }\right\rangle$ is not, at first glance. predicted. but is not impossible to attain in our model.

\section{INTRODUCTION}

The main argument used against a Galactic origin for the gamma-ray bursts (GRBs) is their apparent isotropy on the sky coupled with the observed value of $\left\langle V / V_{\text {max }}\right\rangle$ (Meegan et al. 1992). However, it is dangerous to rule out all Galactic GRB models based on only one combination of two observations. especially when the physics involved in the Galactic models is much easier to believe than that involed in cosmological GRB models. Indeed. the recent evidence for repeat GRBs (Wang \& Lingenfelter 1993: Quashnock \& Lamb 1993a) and a trace of a correlation of GRBs with the Galactic disk (Atteia \& Dezalay 1993: Quashnock \& Lamb 1993b) suggest the necessity of a Galactic GRB model. Hence, the issue here is the likelihood that the popular neutron star (NS) merger model can produce the observed GRBs at cosmological distances compared with a near conspiracy of the distribution and properties of Galactic sources necessary to produce the observed isotropy and $\left\langle V / V_{\max }\right\rangle$.

In this paper. we consider a GRB model based on the accretion of solid bodies onto high-velocity Galactic NSs. This theory predicts several of the observed properties of GRBs. but the high degree of isotropy of GRBs on the sky coupled with the observed value of $\left\langle V / V_{\max }\right\rangle$ is not naturally predicted. However, is not impossible to obtain the observed combination in Galactic GRB models. Two existing ideas on how to accomplish this are 1) a local model involving the nearby spiral structure of the Galaxy plus repeat bursts (Quashnock \& Lamb 1993a. 1993b). and 2) an extended halo model involving high- velocity NSs with a delayed turn on in bursting ( $\mathrm{Li} \&$ Dermer 1992).

Before we go into the details of our model. we would like to point out some problems with the physics involved in the cosmological GRB model based on mergers of pairs of NSs. This model faces the extreme difficulty of obtaining a hard GRB spectrum. and the relatively long duration. but episodic. emission of GRBs. The neutrino emission. necessary to produce a neutrino fireball. is less efficient than the process within a supernova. where the leptons are initially trapped and compressed to a high Fermi level before they escape by diffusion 
with an increase in energy of up to a factor of $10^{2}$. This process will not take place in the NS merger case because the matter is already de-leptonized or transparent. The accretion from the disk formed during the merger onto the central merged object could indeed be episodic and delayed. but the re-leptonization by initial expansion would not then lead to trapping by the relatively slow and turbulent process of alpha disk accretion. If a neutrino fireball were formed. the escape of the thermal radiation. up shifted to gamma rays by the relativistic blue shift of the expanding fireball, is strongly inhibited by the pair opacity and ion dinamical friction. The emission of gamma rays from the relativistic shock (Lorentz factor $\sim 10^{3}$ ) of compact supernovae has proven to be extremely small. Here the total energy of $\sim 10^{49}$ ergs is comparable with that required for cosmological GRBs. and the required Lorentz factor is similar (Colgate 1968: Colgate $\mathcal{S}$ Mcliee 1973; Colgate $\mathcal{S}$ - Petschek 1979). After expansion of the relativistic fireball and the conversion of its initial energy entirely into ultra highly-relativistic nucleons (Lorentz factor of $10^{6}$ ). the reconversion of this nucleon kinetic energy back into hard photons in the ISM, below $100 \mathrm{Mev}$ in photon energy, is extremely inefficient because of the lack of coupling processes. If this energy has to flow through electron processes, then the resulting bremsstrahlung spectrum will be extremely soft. since it must occur in the transparent limit. These difficulties justify a continuing investigation of Galactic GRB models.

\section{THE GALACTIC MODEL}

The Galactic model depends upon the formation of Type II or Ib core collapse supernova (SN) in pre-SN binary systems. We select a typical pre-SN binary of a $10 M_{0}$ companion and a $5 . M$ pre-SN star where the masses were reversed before mass transfer. and are now in a 10-day orbit with $a=0.2 \mathrm{AL}$. We expect that the NS formed in the collapse and SN explosion of the $\bar{J} \mathrm{M}$ star will be ejected at a much higher than average velocity.

High-velocity NSs are created by asymmetric accretion during the SN process from slowly rotating pre-SN stars. Slow rotation occurs because of tidal locking caused by meridional circulation (Tessoul \& Tassoul 1990), as olsserved by Griffin et al. (1993) at an evolution phase long before mass transfer. Herant. Benz \& Colgate (1992) have shown that up to $0.1 \mathrm{~K} \cdot \mathrm{c}$ may be accreted as.mmetrically creating a single large plume in the opposite direction and leading to a recoil velocity greater than $10^{3} \mathrm{~km} \mathrm{~s}^{-1}$. This accretion is $\sim 10^{2}$ times more sensitive to rotation than the NS star itself. and thus rapidly-rotcting NSs are not likely to attain high velocities. while slowly-rotating ones are likely to reach the escape velocity from the Galaxy.

The mass ejected from a $10 M_{-}$secondary star by the impact of the ejecta from the SN is of the order of $\sim 1 M_{0}$ (Wheeler. Lecar \& Mcliee 1975). and $\sim 10^{-5}$ of this mass will be captured into a disk around the NS. based on Nakamura \& Piran (1991) and our own calculations. This mass of $2 \times 10^{28} \mathrm{~g}$ is more than enough to make the planetoids necessary to explain the observed GRB rate. We estimate one such $\mathrm{SN}$ event in the Galaxy per $10^{2}$ years. since binary stars are as common as non binaries. Since there are roughly $10^{3} \mathrm{GRBs}$ per year, then each high-velocity NS+disk system must produce $10^{5}$ events in total. Each Galactic halo burst requires $2 \times 10^{41} \mathrm{ergs}$ (less for local disk models) and so the mass accreted in the lifetime of the system must be $10^{26} \mathrm{~g}$. Since the assumed bursts here are $\sim 10^{3}$ times the Eddington luminosity and the 
mass required eren then is $0.5 \%$ of what is available. this leares ample mass for the soft gamma-ray repeater phase and later the formation of a dynamicallydominant body required in order to gravitationally scatter the smaller bodies both into the NS and out of the system.

Soft gamma-ray repeaters (SGR) will occur when the disk is still largely in a gaseous state. The gas in the accretion disk moves inwards due to the alpha viscosity process, and accumulates at the Alfven radius of the NS. Once the pressure in the "thin" disk exceeds the magnetic pressure from the NS. the gas streams rather suddenly onto the poles of the NS along the magnetic field lines, producing a soft GRB. Such erents occur before the NS has had a chance to move very far from its SN remnant. which w Id explain the observed correlation between SGRs and SN remnants (e.g., Kulwarni \& Frail 1993). The SGR phase terminates due to the thinning of the disk by mass accretion. This is $\sim 10^{3}$ years for $\mathrm{a} \sim 10^{28} \mathrm{~g}$ disk and the Eddington limit. It will be longer depending upon the fraction of "on time" of the repeater and the extent to which the emission exceeds the Eddington limit. The shut-off condition for this alpha phase of accretion is when the disk becomes thin enough so that conrection and frictional heating, necessary to drive the alpha viscosity turbulence. can no longer take place. This corresponds to a thickness of radiative cooling in one rotation period. and hence predicts a threshold thickness of a few to ten $\mathrm{g} \mathrm{cm}^{-2}$. The total mass at $\sim 1 \mathrm{AU}$ is then $10^{27} \mathrm{~g}$, about $10 \%$ of the original mass, and comfortably close to one earth mass. The accretion clisk then cools rapidly and grains condense. the alpha process stops. and the SGR phase ends. There is a long delay before the classical GRB phase begins. due to the need to build up large solid bodies from the grains.

Once cooling has reduced the temperature below the point where molecules and grains can form. a prediction of the time for the formation of a single dynamically-dominant solid body can be made. We assume that the growth of molecules. grains. solid bodies. etc... is proportional to the cross sectional area and a constant virial velocity. For constant average density, the rate of accretion growth for a body of mass $M$ is $d M / d t \propto r^{2}$, where $r$ is the radius of the body. Since $M \propto r^{3}$. the total time to accrete to a radius $r$ becomes $t_{0} \times r / r_{0}$. where $t_{o}$ is the initial sticking of molecules of radius $r_{0}$. The value of $t_{0}$ for molecules at the density of the disk at $\sim 1 A\left[\right.$ (i.e.. $\sim 10 \mathrm{~g} \mathrm{~cm}^{-2}$ ), and a velocity of $10^{4} \mathrm{~cm} \mathrm{~s}^{-1}$ is roughly $\sim 0.1 \mathrm{~s}$. The solid bodies must grow until they reach the size where the gravitational scattering exceeds the collisional cross section. This occurs at roughly $r=30 \mathrm{~km}$. or the start of runaway accretion leading to a single dynamically-dominant solid body. The ratio in size of a molecule to $30 \mathrm{~km}$ is $3 \times 10^{15}$. and so $10^{7}$ years is required. (This is comparable with the time to form the solar system.) The average mass of the solid bodies not accreted into the dominant one is $10^{20} \mathrm{~g}$. This is the starting point of gamma-ray bursts.

The accretion by NSs of solid bodies (Harwit and Salpeter 1973) was considered in detail by Colgate and Petschek (1981: henceforth CPS1) including the distortion. and compression of the body due to the gravity and magnetic field of the NS. In this case. where we have assumed a strong magnetic field for the NS (i.e. $3 \times 10^{12} \mathrm{~g}$ ), the magnetic pressure. $B^{2} / 8 \pi$. is $>\rho v^{2}$ of the accreting matter (where $\rho$ and $v$ are mass density and velocity. respectively) even for a direct impact and including the expected tidal compression ratio of 30 (C'PS1). Thus the cold degenerate matter will enter the field as unstable sheets (CP81), thus transferring the kinetic energy of impact to both the distortion energy of the magnetic field as well as the thermal energy of the plasma. The distortion 
energy of the field will be given up by reconnection. producing the very high energy part of the spectrum by $E_{\text {parallel }}$ acceleration (Colgate 1992). The thermalized matter will settle along the field lines supported by the NS as a constant entropy atmosphere. maintained by convection and field interpenetration. As pointed out in CPS1. the maximum emission rate when the plasma pressure is confined by magnetic field is not the Eddington limit. but instead of the order of $(c / 4)\left(B^{2} / 8 \pi\right)=3 \times 10^{33}$ ergs cm $\mathrm{cm}^{-2} \mathrm{~s}^{-1}$. or a luminosity $<3 \times 10^{46} \mathrm{ergs} \mathrm{s}^{-1}$. A small fraction of this. $10^{-5}$, is sufficient to satisfy a Galactic halo model.

\section{OBSERVATIONAL PREDICTIONS}

Our GRB model makes several observational predictions. Due to the high velocities of the NSs and the delay before GRBs are produced, the correlation with star formation regions in our Galaxy should be weak. No correlation of GRBs with pulsars is expected, since few of the slowly-rotating high-velocity NSs are observable as pulsars, simply because they are rotating so slowly. A correlation of GRBs with the Galactic disk should eventually become obvious after more GRB events are observed. An instrument that is more sensitive than BATSE may help detect such a correlation. and also GRBs from M31.

The maximum GRB luminosity is $\simeq 10^{38} \mathrm{erg} \mathrm{s}^{-1}$ for local Galactic models, and perhaps $10^{3}$ times larger for extended halo models.

The accretion rate of solid bodies by a given NS (and thus the resulting GRB rate) increases rapidly once a dynamically-dominant solid body is formed. probably via a phase of runaway accretion. Afterwards, the GRB rate falls off exponentially as the smaller solid bodies are depleted via being scattered into the NS, and (mostly) very large orbits.

The growth of the solid bodies continues with time as the NS moves away from the Galactic plane. Since. as pointed out above. the radii of the solid bodies grow in proportion to time, the GRB events that occur farther out in the Galactic halo involve. on average, more massive solid bodies. Assuming that the maximum luminosity of GRBs is less than proportional to the mass. because of either an upper-limiting maximum emission rate (e.g., the Eddington limit) or diffusion processes, then GRBs that originate farther out in the Galactic halo have, on average. a longer duration. Since the flux received from distant GRB rents is smaller. we predict that longer bursts are. on average, fainter. Such bursts should also be softer, because larger mass implies greater thermalization.

The spectrum of total GRB energies from a given GRB source is like the mass spectrum of small solid bodies that form via accretion. The decrease in the number of bodies as accretion occurs is $d N / d t \propto-. V r^{2}$, or $d N / d t \propto-N d M / d t$. Since $N \propto M^{-1}$, we find $d N / d M \propto M^{-1}$. Since the total energy of a GRB, $E$. is proportional to $M$. then $d N / d E \propto E^{-1}$.

Repeat bursts are expected as a result of the tidal fracturing of the small solid bodies during close encounters with the dynamically-dominant body. Repeat bursts are not likely to be of the same total energy, since the masses of the solid bodies depend so strongly on their radii (i.e., $M \propto r^{3}$ ). There should be a broad spectrum of repeat times. and the fainter bursts (small solid bodies without enough "oomph" to reach the maximum luminosity) may both precede and/or follow the main burst.

In summary, we believe that this model makes many predictions concerning stars. supernova, neutron stars, planetary systems, and gamma-ray bursts that are exciting. 


\section{REFERENCES}

1. J. -L. Atteia and J. -P. Dezalay: Ad.A 274. L1 (1993).

2. S. A. Colgate. C'anadian Journal of Phrsics 46. 476 (1968).

3. S. A. Colgate. Proceedings of the Los Alamos Workshop on Gamma-Ray Bursts. edited by C. Ho et al. (Cambridge University Press. 1992), p. 75.

4. S. A. Colgate and A. G. Petschek. ApJ 229, 682 (1979).

5. S. A. Colgate and A. G. Petschek, ApJ 248, 771 (1981).

6. S. A. Colgate and C. R. Mcliee. ApJ 181, 903 (1973).

7. R. E. M. Griffin et al.. A\&A 274. 225 (1993).

8. M. Harwit and E. E. Salpeter, ApJ 186, L37 (1973).

9. M. Herant. W. Benz and S. A. Colgate, ApJ 395, 642 (1992).

10. S. R. Kulkarni and D. A. Frail. Nature 365, 33 (1993).

11. H. Li and C. D. Dermer. Nature 359. 514 (1992).

12. C. A. Meegan et al.. Nature 355, 143 (1992).

13. T. Nahamura and T. Piran. ApJ 382. L81 (1991).

14. J. M. Quashnock and D. Q. Lamb, MNRAS . in press (1993a).

15. J. M. Quashnock and D. Q. Lamb. MNRAS . in press (1993b).

16. J. -L. Tassoul and M. Tassoul. ApJ 359, 15 (1990).

17. V. C. Wang and R. E. Lingenfelter, ApJ 416. L13 (1993).

18. J. C. Wheeler, M. Lecar and C. F. McIiee. ApJ 200, 145 (1975). 

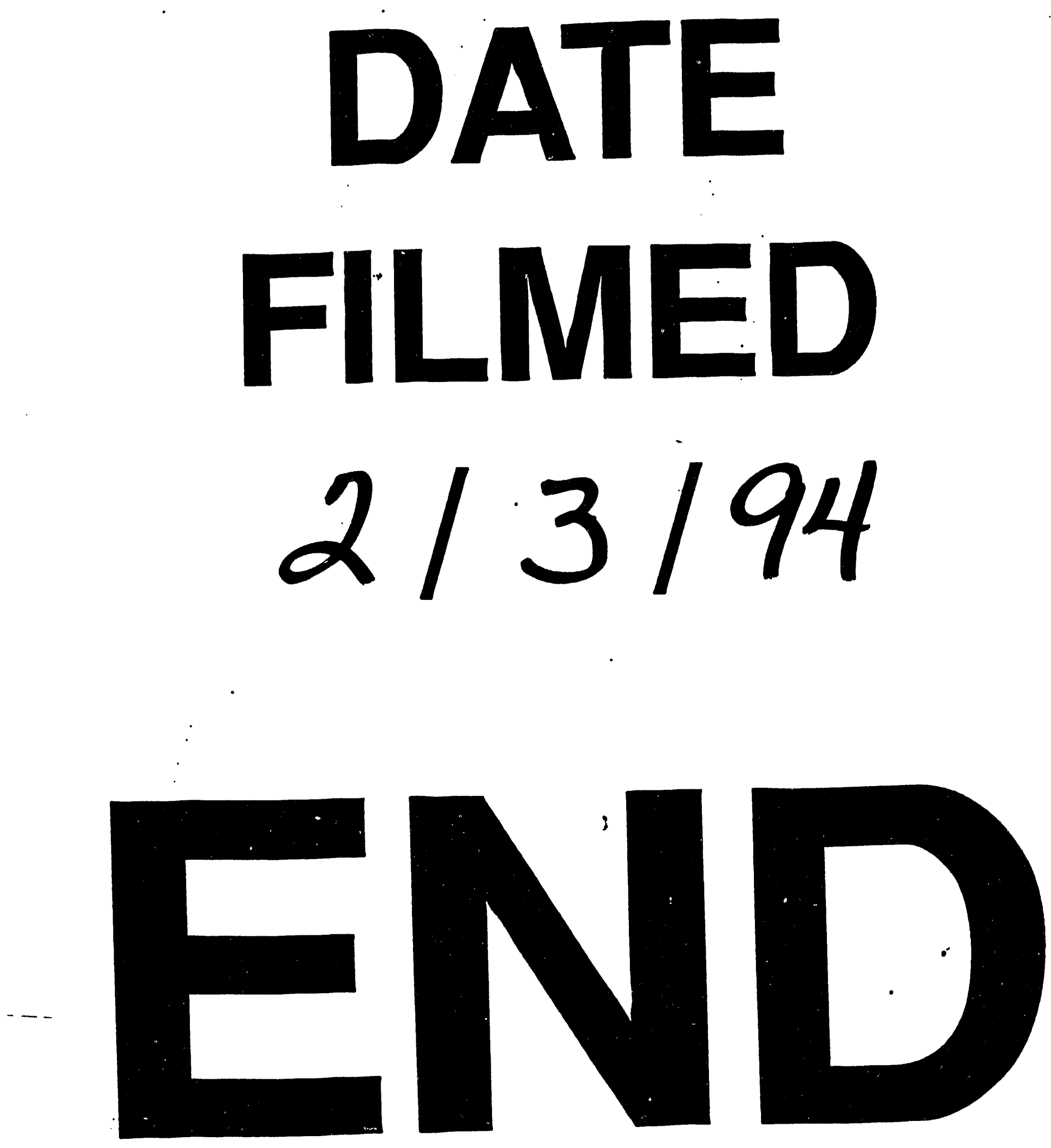
